\title{
Logarithmic running of 't Hooft-Polyakov monopole to dark energy
}

\author{
M. S. EI Naschie \\ Dept. of Physics, University of Alexandria, Alexandria, Egypt \\ Email address: \\ Chaossf@aol.com
}

\section{To cite this article:}

M. S. El Naschie. Logarithmic Running of 't Hooft-Polyakov Monopole to Dark Energy. International Journal of High Energy Physics. Vol. 1, No. 1, 2014, pp. 1-5. 10.11648/j.ijhep.20140101.11

\begin{abstract}
The paper presents a particle physicists' interpretation of the mathematical abstract concept of a five dimensional empty set as the source of dark energy and dark matter. It turns out that the simplest alternative physical interpretation at least from the view point of the GUT unification of fundamental interaction is the theoretically well established but experimentally never found yet ' $t$ Hooft-Polyakov magnetic giant monopole with the predicted huge mass of ten to the power of $16 \mathrm{Gev}$. In fact it will be shown here using exact renormalization equations that running the preceding energy logarithmically leads to a prediction of the ordinary and the total dark energy density of the cosmos in complete agreement with our earlier result $\mathrm{E}(\mathrm{O})=\mathrm{mc}^{2} / 22$ and $\mathrm{E}(\mathrm{D})=\mathrm{mc}^{2}(21 / 22)$ based on the afore mentioned set theoretical concepts as well as with all the relatively recent cosmological measurements. The decisive steps in the present derivation consists of two realizations. First and to our deepest surprise and delight, $\mathrm{E}=\gamma \mathrm{mc}^{2}=\mathrm{mc}^{2}$ is actually a unification formula uniting classical, relativistic and quantum mechanics where $\gamma=1$ corresponds to a $100 \%$ energy density. Second and also not expectedly, the logarithmic running of 't Hooft-Polyakov's monopole energy leads to a reduction factor $\gamma=1 / \lambda$ where $\lambda$ $=\frac{1}{2} \ln \frac{\mathrm{M}(\text { monopole })}{\mathrm{m} \text { (electron) }}=22.18033989$, in full agreement with our previous results using entirely different approaches. Finally the results are validated using 't Hooft's dimensional regularization $D=4-\epsilon$ by setting $\epsilon=2 \emptyset^{5}$ where $\emptyset^{5}$ is Hardy's quantum entanglement and $\phi=2 / \sqrt{5}+1$.
\end{abstract}

Keywords: Dark energy, Grand Unification, Giant 'T Hooft-Polyakov Monopole,

Quantum Relativity Renormalization Equations, Fractal Spacetime, Quantum Field Theory, Super Symmetry, Dark Matter, Planckton, 'T Hooft Renormalization

\section{Introduction}

The major challenge of explaining that our universe is neither static nor simply expanding with a decreasing rate as it was thought for a considerable time but in fact suffers an increased expansion was tackled in numerous recent publications [1-18]. Thus we have offered in the last two years several alternative theories all leading virtually to precisely the same quantative and qualitative results, namely that the ordinary measurable energy density $\mathrm{E}(\mathrm{O})$ is given by

$$
\begin{aligned}
& \mathrm{E}(\mathrm{O})=\left(\phi^{5} / 2\right)\left(\mathrm{mc}^{2}\right) \\
& \simeq \mathrm{mc}^{2} / 22
\end{aligned}
$$

where $\mathrm{m}$ is the mass, $\mathrm{c}$ is the speed of light, $\phi^{5}$ is Hardy's generic probability of quantum entanglement $[6,12]$ and $\phi=2 /(1+\sqrt{5})$. Consequently the rest which we refer to for simplicity as dark energy but really mean dark energy as well as dark matter must be [19]

$$
\begin{aligned}
& \mathrm{E}(\mathrm{D})=\left(1-\phi^{5} / 2\right)\left(\mathrm{mc}^{2}\right) \\
& =\left(5 \phi^{2} / 2\right)\left(\mathrm{mc}^{2}\right) \\
& \simeq \mathrm{mc}^{2}(21 / 22)
\end{aligned}
$$

It is needless to reiterate that $E(D)$ could never be measured directly until this point of time but its existence is inferred from the increased rather than decreased rate of cosmic expansion as well as several observed astronomical anomalies [1-5]. It then turned out that $\mathrm{E}(\mathrm{O})$ is the energy stored in a five dimensional zero set volume modeling the quantum particle while $E(D)$ is the energy stored in the five dimensional volume of the empty set modeling the quantum wave [10-12]. This line of reasoning may be labeled 
transfinite set theoretical-topological resolution of dark energy [9-13]. Another line of attack on this problem was undertaken using geometrical reasoning based upon the properties of real physical spacetime akin to that proposed by Cartan and Einstein as influenced by the pioneering work of the brothers Cosserat [9,20].

In the present work we want to utilize more traditional particle physicists' conceptions in solving this problem and bring it nearer to the understanding of conventional high energy physics and quantum field theory [20-30]. This possibility was indeed realized via a GUT scenario based upon 't Hooft-Polyakov giant monopole [31] as will be explained in the next section.

\section{Assembling the Fundamental Equations Needed for the Analysis}

Central to our present analysis are a few fundamental equations revolving around a transfinitely exact version of the logarithmic renormalization equations of the strong interaction and grand unification [21]. In the transfinite form these otherwise quite complex equations take an unheard of simplicity [22]. For GUT unification for instance the fundamental equation $[21-25,32]$

$$
\bar{\alpha}_{\mathrm{u}}=\bar{\alpha}_{3}+\bar{\alpha}_{4}+\frac{1}{\mathrm{~N}} \ln \frac{\mathrm{M}_{\mathrm{u}}}{\mathrm{M}_{\mathrm{o}}}
$$

becomes

$$
\begin{aligned}
& \bar{\alpha}_{\mathrm{u}}(\mathrm{GUT})=\bar{\alpha}_{3}+\bar{\alpha}_{4}+\frac{1}{\mathrm{~N}} \ln \frac{10^{16} \mathrm{Gev}}{\mathrm{M}_{z}} \\
& =(9+1)+(1)\left[\ln \left(\frac{10^{16} \mathrm{Gev}}{91 \mathrm{Gev}}\right)\right] \\
& =10+32.330501 \mathrm{~N} \\
& =42.33 \\
& \simeq 42
\end{aligned}
$$

where $10^{16} \mathrm{Gev}$ is the grand unification energy scale, $\mathrm{M}_{z}=91 \mathrm{Gev}$ is the mass of the $z^{0}$ particle and $\bar{\alpha}_{3}=9$ and $\bar{\alpha}_{4}=\bar{\alpha}_{\mathrm{QG}}=1$ are the inverse coupling of the strange interaction and the coupling of quantum gravity respectively $[11,12,18]$. We note the proximity of $\bar{\alpha}_{u} \simeq 42.33$ to the exact value found using various methods $\bar{\alpha}_{u}=42.36067977$ [26,27]. It is instructive before going any further to consider the case of super symmetric GUT [29-32]. In this case all that changes is that $\mathrm{N}=2$ instead of 1 which is the minimal case of a single super symmetric partner to each fermion or Boson. Inserting one finds

$$
\begin{aligned}
& \bar{\alpha}_{\mathrm{u}}(\text { super symmetric GUT })=10+\left(\frac{1}{2}\right)(32.33) \\
& \cong 10+16.18 \\
& \simeq 26
\end{aligned}
$$

in full agreement with all results reported in the literature [24-27]. More over we note that $\bar{\alpha}_{u} \cong 26$ is the same value found when gravity is involved which proves the point observed some time ago, namely that super symmetry already implies quantum gravity whether gravity is involved explicitly or not [21-27]. What may be less well known in the field is the fact that probing the Planck scale leads also automatically to the quantum gravity result as may be demonstrated in the following analysis in which we take $M_{u}$ to be the Planck mass, i.e. Planckton with a mini black hole mass $10^{19} \mathrm{Gev}$ and replace $\mathrm{M}_{z}$ by the electron scale $\mathrm{m}_{\mathrm{c}}=$ $0.511 \mathrm{mev}[21-25,33]$. Inserting one finds

$$
\begin{aligned}
& \bar{\alpha}_{\mathrm{u}}(\text { Planck })=\left(\bar{\alpha}_{3} \simeq 0\right)+\left(\bar{\alpha}_{4}=1\right)+\left(\frac{1}{2}\right) \ln \frac{10^{19} \mathrm{Gev}}{(0.511)(10)^{-3} \mathrm{Gev}} \\
& =1+\frac{1}{2} \ln \left(10^{22} / 0.511\right) \\
& =1+\frac{1}{2}(51.32825773) \\
& =1+25.664 \\
& \simeq 26.664
\end{aligned}
$$

Note that the correct result was found but only after setting $\mathrm{N}=2$ as well as the obvious values $\bar{\alpha}_{4}=\bar{\alpha}_{\mathrm{QG}}=1$ and $\bar{\alpha}_{3}=\infty$ which means $\bar{\alpha}_{3}=0$ [24-27]. Encouraged by these results we could apply the entire previous mathematical machinery developed initially by the pioneering efforts of 't Hooft, Kadanof, Wilson, Fisher and Gross to find the exact value of the most basic and fundamental constants of nature, namely $\bar{\alpha}_{\mathrm{o}} \cong 137$. Without going into detail this is found to be [24-27]

$$
\bar{\alpha}_{\mathrm{o}}=\left(\bar{\alpha}_{2}+\bar{\alpha}_{3}+\bar{\alpha}_{4}\right)+\left(\bar{\alpha}_{1}\right)(1 / \phi)
$$

where $\quad \bar{\alpha}_{1}=60, \quad \bar{\alpha}_{2}=30, \quad \bar{\alpha}_{3}=9, \quad \bar{\alpha}_{4}=1$, $1 / \phi=1+\phi=1.618033989$ and $\phi=(\sqrt{5}-1) / 2$. Inserting we find

$$
\begin{aligned}
& \bar{\alpha}_{\mathrm{o}}=\mathrm{R}^{(4)}(1 / \phi)^{5-1} \\
& =(20)(1 / \phi)^{4} \\
& =137+\mathrm{k}_{\mathrm{o}} \\
& =137+\phi^{5}\left(1-\phi^{5}\right) \\
& =137.082039325
\end{aligned}
$$

exactly as should be $[26,27]$ where $\mathrm{k}=\phi^{3}\left(1-\phi^{3}\right), \mathrm{k}_{\mathrm{o}}=$ $\mathrm{k}_{\mathrm{o}}=\phi^{5}\left(1-\phi^{5}\right), \phi=1 /(1+\sqrt{5})$ and $\phi^{5}$ is Hardy's quantum entanglement probability. Now we are in a position to tackle the problem at hand regarding dark energy [5-36].

\section{Coupling at the Threshold of GUT and Dark Energy Density}

Let us apply our renormalization equation to the situation when the unification scale is that of the 't Hooft-Polyakov GUT monopole and the reference scale is that of 
electromagnetism. Thus we are involving a giant monopole with $\mathrm{M} \simeq 10^{16} \mathrm{Gev}$ indirectly with the smallest magnetic charge possible associated with normal electric current, i.e. the electron. Setting $N=2$ and observing that $\bar{\alpha}_{3}$ and $\bar{\alpha}_{4}$ must be zero, one finds

$$
\begin{aligned}
& \bar{\alpha}_{\mathrm{u}}=0+\frac{1}{2} \ln \left[\frac{10^{16} \mathrm{Gev}}{(0.511)(10)^{-3} \mathrm{Gev}}\right] \\
& =\frac{1}{2}\left(\ln \frac{10^{19}}{0.511}\right) \\
& =\frac{1}{2}(44.42050246) \\
& \cong 22.2 \\
& \simeq 22+\mathrm{k}
\end{aligned}
$$

In other words $\bar{\alpha}_{\mathrm{u}}=(26+\mathrm{k})-4=22+\mathrm{k} \quad$ could naturally be interpreted as the compactified dimensions of the bosonic string space of the Nambu-Veneziano strong interaction model [15] bringing two instructive tautological equations $[9,19]$

$$
\begin{aligned}
& \sum_{1}^{4} \bar{\alpha}_{\mathrm{u}}=60+30+9+1 \\
& =\left(\mathrm{D}^{(10)}\right)^{2} \\
& =100
\end{aligned}
$$

and

$$
\begin{aligned}
& \mathrm{D}(26)+\bar{\alpha}_{2}+44 \\
& =100
\end{aligned}
$$

to interact with the basically cosmic measurement of energy expressed in a percentage [19]

$$
\begin{aligned}
& E_{\text {total } \%}=\left(\frac{1}{2} 2+\mathrm{k}\right) \%+(22+\mathrm{k}) \%+73.3116292 \% \\
& =(4.568497187)+(22+\mathrm{k})+(73.31116292) \\
& =100 \%
\end{aligned}
$$

Thus the following two energy densities sum up the most important conclusions which we can draw from the preceding analogy:

(a) $\mathrm{E}(\mathrm{O})=\mathrm{mc}^{2} /\left(\bar{\alpha}_{\mathrm{u}}\right)=\mathrm{mc}^{2} /(22+\mathrm{k})$

(b) $\mathrm{E}(\mathrm{D})=1-\mathrm{E}(\mathrm{O})$

$$
\begin{aligned}
& =\left(\frac{1}{1+\frac{1}{21+\mathrm{k}}}\right) \mathrm{mc}^{2} \\
& =\mathrm{mc}^{2}[(21+\mathrm{k}) /(22+\mathrm{k})]
\end{aligned}
$$

We note that $E(D)$ clearly marks the state when our essentially fractal universe [34,35] shrinks to almost one dimension. The exact value is actually one plus the ratio one to what is left from the $26+\mathrm{k}$ when we allow for the 5 dimensions of de Sitter which is the precursor for the ten dimensionality of superstrings and the eleven dimensions of super gravity and Witten's M-theory. Of course it all started with Kaluza-Klein's 5D space and it may be at least a source of bemusement to ponder if there are truly non-trivial deep connections between the sum of all internal and space dimensions involving E8E8 and Einstein's $D^{(4)}$ on the one side and the sum of all inverse fundamental constants on the other side, which leads to $\mathrm{D}^{(5)}$

$$
\begin{aligned}
& \frac{|\mathrm{E} 8 \mathrm{E} 8|+\mathrm{D}^{(4)}}{\sum_{1}^{4} \bar{\alpha}_{\mathrm{i}}}=\frac{496+4}{100} \\
& =\mathrm{D}^{(5)} \\
& =5
\end{aligned}
$$

These and other relations will be dealt with may be in future publications. However the author could not resist communicating yet another derivation of $\mathrm{E}(\mathrm{O})$ based on Newton's kinetic energy with a Weyl scaling made of the ratio of $\mathrm{D}(4)$ to the difference between the exceptional manifold with $\mathrm{D}=(5)\left(\bar{\alpha}_{\mathrm{o}}\right)=548$ dimensions and the Heterotic manifold with 504 dimensions which leads to 4 excess dimensions leading to

$$
\mathrm{E}_{\mathrm{N}}=\frac{\mathrm{m}}{2}\left(\frac{4}{548-540}\right)(\mathrm{v} \rightarrow \mathrm{c})^{2}=\frac{1}{2} \mathrm{mc}^{2}\left(\frac{4}{44}\right)=\mathrm{mc}^{2} / 22
$$

In fact $548-540=44$ could be interpreted as $|\mathrm{SO}(10)|=$ 45 of GUT minus the single photon of Einstein, i.e. $45-1$ $=44[28,29]$ apart of being the 44 components of a Vierbein [36]. Note also that 44 are the degrees of freedom of a massless graviton or pure gravity in $\mathrm{d}=11$ Witten M-theory [36].

\section{Could $E=m c^{2}$ be a Quantum Gravity Formula - A Discussion beyond Convention}

Both the P-Adic representation of $\bar{\alpha}_{0}=137$ and more general Witten's T-duality $[9,36]$ lead us to consider the reality of the unit interval physics and that $\mathrm{E}=\gamma \mathrm{mc}^{2}=\mathrm{mc}^{2}$ states clearly that $\gamma=1$ means $\bar{\alpha}_{\mathrm{QG}}=1$. Conversely $\bar{\alpha}_{\mathrm{u}}=22+\mathrm{k}$ means that $\gamma=1 / \bar{\alpha}_{\mathrm{u}}=1 /(22+\mathrm{k})$ and therefore the energy density is

$$
\mathrm{E}=\mathrm{m}^{2} /(22+\mathrm{k})=\mathrm{E}(\text { ordinary })
$$

In other words Witten's T-duality applies to the entire cosmos and could be interpreted as saying that Einstein discovered quantum gravity long before quantum mechanics was discovered. Was it genius, good luck or simply a case of Kostler sleep walking! That we will probably never know for sure but the fact that

$$
\mathrm{E}(\mathrm{O})+\mathrm{E}(\mathrm{D})=\mathrm{mc}^{2}
$$


where $\mathrm{E}(\mathrm{O})=\left(\phi^{5} / 2\right) \mathrm{mc}^{2}$ and $\mathrm{E}(\mathrm{D})=\left(5 \phi^{2} / 2\right) \mathrm{mc}^{2}$ is indeed startling particularly when we realize that $\phi^{5}$ is Hardy's experimentally verified probability of quantum entanglement [6] corresponding to the volume of the five dimensional zero set which models the quantum particle and $5 \phi^{2}$ corresponds to the five dimensional volume of the empty set which models the quantum wave [10].

\section{Conclusion}

We showed in previous publications that dark energy could be determined from Rindler's spacetime [7,13]. On the other hand the Rindler wedge is usually considered a toy model for a black hole. Consequently this is by no means a toy model as is usually supposed, but a real model for the cosmos $[7,13]$. In a sense when Perlmutter, Schmidt and Rees measured the accelerated cosmic expansion [1-6] they were quasi-Rindler observers $[7,13]$. In the present work we demonstrated that it is sufficient to look at GUT unification, i.e. $10^{16} \mathrm{Gev}$ using the giant form of ' $\mathrm{t}$ Hooft-Polyakov monopole [30] to reach the same not only qualitative but also quantative conclusions which we draw regarding dark energy from a fully Planck scale black hole unification, i.e. $10^{19} \mathrm{Gev}$ [28]. The simplest ultimate validation of the present work comes however from noticing that gravity must have a profound effect on gauge forces coupling and that could be accounted for exactly via 't Hooft's dimensional regularization [37,38]. This is simply achieved by setting $\in=4-\mathrm{D}$ equal to $2 \phi^{5}=\mathrm{k}$ and not letting $\in \rightarrow 0$. That way one finds $\mathrm{E}(\mathrm{dark})=[(4-\mathrm{k}) / 4] \mathrm{mc}^{2}$ $\simeq \mathrm{mc}^{2}(21 / 22)$ exactly as expected. The details of this analysis will be given in a forthcoming work.

\section{Acknowledgement}

The Author thanks an anonymous referee for the constructive suggestions which helped to improve the present paper.

\section{References}

[1] A.G. Riess et al, Observation evidence from supernova for an accelerating universe and a cosmological constant. The Astronomical Journal. Vol. 116, pp. 1009, 1998. Doi: $10.1086 / 300499$.

[2] S. Perlmutter et al, Supernova cosmology project collaboration. "Measurement of omega and lambda from 42 high-redshift supernova. The Astrophysical Journal, Vol. 517, No. 2, 1999, pp. 565-585.

[3] C.L. Bennett, WMAP Collaboration. Astrophys. J. Suppl,Vol. 148, No. 1, 2003, [astro-ph/0302207].

[4] B.P. Schmidt et al, The distance to five type II supernova using the expanding photosphere method and the value of Ho. The Astrophys. J., Vol. 432, 1994, pp. 42-48.
[5] T. Padmanabhan, Dark Energy: The cosmological challenge of the millennium. arXiv: astro-ph/0411044V1, 2 Nov 2004.

[6] M.S. El Naschie, A resolution of cosmic dark energy via a quantum entanglement relativity theory. J. Quantum Info. Sci., Vol. 3, No. 1, 2013, pp. 23-26.

[7] M.S. El Naschie, A Rindler-KAM spacetime geometry and scaling the Planck scale solves quantum relativity and explains dark energy. Int. J. of Astron. and Astrophys.Vol. 3, No. 4, 2013, pp. 483-493.

[8] M.S. El Naschie, From Yang-Mills photon in curved spacetime to dark energy density. J. Quantum Info. Sci., Vol. 3, No. 4, 2013, pp. 121-126.

[9] M.S. El Naschie and M.A. Helal, Dark energy explained via the Hawking-Hartle quantum wave and the topology of cosmic crystallography. Int. J. Astron. \& Astrophys, Vol. 3, 3, 2013, pp. 318-343.

[10] M.S. El Naschie, "Topological-geometrical and physical interpretation of the dark energy of the cosmos as a 'halo' energy of the Schrödinger quantum wave." J. Mod. Phys., Vol. 4 , No. 5, 2013, pp. 591-596.

[11] L. Marek-Crnjac and Ji-Huan $\mathrm{He}$, An invitation to El Naschie's theory of Cantorian spacetime and dark energy. Int. J. of Astron. \& Astrophys., Vol. 3, 2013, pp. 464-471.

[12] M.A. Helal, L. Marek-Crnjac and Ji-Huan He, The three page guide to the most important results of M.S. El Naschie's research in E-infinity and quantum physics and cosmology. Open J. Microphys., Vol. 3, No. 4, 2013, pp. 141-145.

[13] M.S. El Naschie, Experimentally based theoretical arguments that Unruh's temperature, Hawkings's vacuum fluctuation and Rindler's wedge are physically real. American J. of Modern Phys., Vol. 2, No. 6, 2013, pp. 57-361.

[14] L. Marek-Crnjac, Modification of Einstein's $\mathrm{E}=\mathrm{mc}^{2}$ to $\mathrm{E}=$ $1 / 22$ mc2. American J. Modern Phys., Vol. 2, No. 5, 2013, pp. 255-263.

[15] L. Marek-Crnjac and M.S. El Naschie, Chaotic fractal tiling for the missing dark energy and Veneziano model. Appl. Math., Vol. 4, No. 11B, 2013, pp. 22-29.

[16] Ji-Huan He and L. Marek-Crnjac, Mohamed El Naschie's revision of Albert Einstein's $\mathrm{E}=\mathrm{mc}^{2}$ : A definite resolution of the mystery of the missing dark energy of the cosmos. Int. J. Modern Nonlinear Sci. \& Application., Vol. 2, No. 1, 2013, pp. 55-59.

[17] M.S. El Naschie, Dark energy via quantum field theory in curved spacetime. J. Mod. Phys. \& Appli. Vol. 2, 2014, pp.

[18] Ji-Huan He and L. Marek-Crnjac, The quintessence of El Naschie's theory of fractal relativity and dark energy. Fractal Spacetime \& Noncommutative Geometry in Quantum \& High Energy Phys., Vol. 3, No. 2, 2013, pp. 130-137.

[19] M.S. El Naschie, A unified Newtonian-relativistic quantum resolution of the supposedly missing dark energy of the cosmos and the constancy of the speed of light. Int. J. Modern Nonlinear Sci. \& Application., Vol. 2, No. 1, 2013, pp. 43-54.

[20] F. Hehl, Space-Time as Generalized Cosserat Coninuum. In "Mechanics of Generalized Continua". Editor E. Kronev., Springer Verlag, Berlin, 1968. pp. 347-349. 
[21] M.S. El Naschie, Transfinite harmonization by taking the dissonance out of the quantum field symphony. Chaos, Solitons \& Fractals, Vol. 36, No. 4, 2008, pp. 781-786.

[22] M.S. El Naschie, Extended renormalization group analysis for quantum gravity and Newton's gravitation constant. Chaos, Solitons \& Fractals, Vol. 35, No. 3, 2008, pp. 425-431.

[23] M.S. El Naschie, Asymptotic freedom and unification in a golden quantum field theory. Chaos, Solitons \& Fractals, Vol. 36, No. 3, 2008, pp. 521-525.

[24] E. Goldfain, Renormalization group and the emergence of random fractal topology in quantum field theory. Chaos, Solitons \& Fractals, Vol. 19, No. 5, 2004, pp. 1023-1030.

[25] M.S. El Naschie, Towards a quantum golden field theory. Int J. of Nonlinear Sci. \& Numerical simulation., Vol. 8, No. 4, 2007, pp. 477-482.

[26] M.S. El Naschie, A review of E-infinity and the mass spectrum of high energy particle physics. Chaos, Solitons \& Fractals, Vol. 19, No. 1, 2004, pp. 209-236.

[27] M.S. El Naschie, The theory of Cantorian spacetime and high energy particle physics (an informal review). Chaos, Solitons \& Fractals, Vol. 41, No. 5, 2009, pp. 2635 - 2646.

[28] M.S. El Naschie, SO (10) grand unification in a fuzzy setting. Chaos, Solitons \& Fractals, Vol. 32, No. 3, 2007, pp. 958-961.

[29] M.S. El Naschie, SU(5) grand unification in a transfinite form. Chaos, Solitons \& Fractals, Vol. 32, No. 2, 2007, pp. 370-374.
[30] P. Langacker, Grand unification. Scholarpedia 7(10):11419, 2012. (See in particular Fig. 3).

[31] D. Gross and M.J. Perry, Magnetic Monopoles in Kaluza-Klein Theories. Nuclear Phys. Vol. B226, 1983, pp. $29-48$.

[32] M.S. El Naschie, Bounds on the number of possible Higgs particles using grand unification and exceptional Lie groups. Chaos, Solitons \& Fractals, Vol. 37, No. 3, 2008, pp. 633-637.

[33] J.R. Ellis, Particle physicists' candidates for dark matter. Phil. Trans. R. Soc. London A, Vol. 320(1556), 1986, pp. 475-485.

[34] Yong Tao, The validity of dimensional regularization method on fractal spacetime. Journal of Appl. Math. 2013. arXiv: http://dx.doi.org/10.1155/2013/308691.

[35] A. Elokaby: Knot wormholes and the dimensional invariant exceptional Lie groups and Stein sspace hierarchies. Chaos, Solitons \& Fractals, Vol. 41, No. 4, 2009, pp. 1616 - 1618.

[36] M.J. Duff, The World in Eleven Dimensions. Inst. of Phys. Publications, Bristol 1999.

[37] G. 't Hooft, A Confrontation With Infinity. In "Frontiers of Fundamental Physics" 4. Editors B. Sidharth and M. Altaisky. Kluwer-Plenum, New York (2001), pp. 1-12.

[38] M.S. El Naschie, 't Hooft's dimensional regularization implies transfinite Heterotic string theory and dimensional transmutation. In "Frontiers of Fundamental Physics" 4. Editors B. Sidharth and M. Altaisky. Kluwer-Plenum, New York (2001), pp. 81-86. 